\title{
Diskussion
}

\author{
Mechthild Rumpf, Eva Senghaas-Knobloch
}

\section{»Dem Reich der Freiheit werb' ich Bürgerinnen«}

\section{Ute Gerhard zum 65. Geburtstág}

(Louise Otto)

Das Cornelia Goethe Centrum für Frauenstudien und die Eyforschung der Geschlechterverhältnisse der Johann Wolfgang GoetheUniversität Frankfurt/M. veranstaltete am 13./14. Februar 2004 eine internationale Konferenz zum Thema "Das Jahrhundert des Feminismus«, um damit seine Mentorin Ute Gerhard zu ihrem 65. Geburtstag zu ehren. ${ }^{1}$

Seit 1987 lehrt und forscht Ute Gerhard als bundesweit erste Frauenforschungs-Professorin für Soziologie mit dem Schwerpunkt Frauenarbeit und Frauenbewegung am Fachbereich Gesellschaftswissenschaften der Johann Wolfgang Goethe-Universität in Frankfurt/M. Dort existierte bereits seit den 1970er Jahren eine lebendige und intellektuell anregende inner- und außeruniversitäre community, die um Debatten feministischer Theorie, Wissenschaft und Praxis kreiste. So war der Weg nach Frankfurt kein einsamer Start für die aus Bremen berufene neue Professorin. Ute Gerhard ist Mitbegründerin und war bis vor kurzem geschäftsführende Direktorin der seit 1997 bestehenden interdisziplinären, zentralen Forschungseinrichtung Cornelia
Goethe Centrum für Frauenstudien und die Erforschung der Geschlechterverhältnisse. Anlässlich der Namensgebung oder "Taufe" des Centrums hatte sie an den berühmten Essay "A Room of One`s Own" (1928) von Virginia Woolf erinnert, in dem der "Gedanke an die Schwestern berühmter Männer bereits ein provokantes Leitmotiv" darstellt, "das immer wieder sehr kunstvoll und einnehmend aufgegriffen und poetisch weitergesponnen wird." Sie verband damit die bereits existente Verpflichtung des Centrums, die Geschlechterperspektive in die verschiedenen wissenschaftlichen Disziplinen »hineinzutragen“, "Aufklärung und Kritik sowie das Prinzip Geschwisterlichkeit wissenschaftlich einzuüben und zu praktizieren" und dies über die Enge des sprichwörtlich "grauen" Elfenbeinturms in praktischer Absicht auch hinauszutragen (Gerhard 2000).

Die Forschungsprojekte, Lehrprogramme, internationalen Kooperationen, Workshops und Konferenzen sowie das Graduiertenkolleg des Centrums dokumentieren eindrucksvoll diese anspruchsvolle Selbstverpflichtung und den kreativen

1 Vorbereitet und koordiniert wurde die Konferenz von Ulla Wischermann und Anja Weckwert. Ein Tagungsband ist in Vorbereitung. Der hier präsentierte Beitrag basiert in Teilen auf dem Eröffnungsvortrag von Eva Senghaas-Knobloch "Menschenrechte und fürsorgliche Praxis in einer zerklüfteten Welt - feministische Maßstäbe». (Auszüge ihres Vortrags wurden am 24. 2. 2004 in der Frankfurter Rundschau dokumentiert.) Mechthild Rumpf hat Passagen ihres vorbereiteten Statements für die Abschlussdiskussion der Konferenz in erweiterter Form beigesteuert. Die jeweils vollständigen Konferenzbeiträge werden in dem geplanten Tagungsband publiziert. 
Geist, der dort weht. In jüngerer Zeit standen die Themen "Islam und $\mathrm{Ge}$ schlechterverhältnisse «, "Employment and Women's Studies: The Impact of Women's Studies Training on Women's Employment in Europe" und "Working and Mothering. Social Practices and Social Policies" im Vordergrund der Forschungspraxis. $^{2}$

Wer längere Zeit am Centrum verweilen konnte, spürt, wie das Interesse an der gemeinsamen Sache und das "Prinzip Geschwisterlichkeit" gegen alle Zwänge des Wissenschaftsbetriebs und gegen alle Konkurrenzmechanismen Bestand haben. Der Beitrag von Ute Gerhard zu dieser Atmosphäre, ihre ansteckende Begeisterungsfähigkeit und nie nachlassende wissenschaftliche Neugier, ihre Kritik und Kreativität wurden im Rahmen ihrer akademischen Verabschiedung auf fantasievolle Weise gewürdigt.

Der weite und interdisziplinäre Denkhorizont, in dem Ute Gerhards Studien angesiedelt sind, verdankt sich einem Doppelstudium von Rechtswissenschaft und Soziologie sowie einem Begleitstudium im Fach Geschichte. Ihre Sensibilität für die Alltagspraxis von Frauen, für deren Bedürfnisse, Unrechtserfahrungen, Ansprüche und Utopien beruht jedoch nicht nur auf sozialwissenschaftlicher Erfahrung und historisch informiertem Wissen, sondern ist auch Teil der eigenen biografischen Wirklichkeit. Die Vehemenz, Gleichheit ohne Angleichung an die "Mannesstellung" als Zukunftsprojekt zu denken, ist auch in ihrer eigenen Erfahrung als Mutter von drei Töchtern verwurzelt. Diese komplexe Wissensbasis inspiriert alle ihre Anstrengungen, die ins "Dunkel des Hauses" (Arendt) verbannten
Tätigkeiten fürsorglicher Praxis in ihrer gesellschaftlichen Relevanz und ihrem humanen Potenzial für den öffentlichen Diskurs sichtbar zu machen und die Fragen des guten Lebens als gesellschaftliche und nicht als private - Frauen auf den Leib geschriebene - Fragen zu behandeln.

Ihre durch journalistische Tätigkeiten in den 1960er Jahren eingeübte Liebe zur Architektur eines Textes wirkt bis heute nach. Die Sorgfalt gegenüber Texten haben viele Kolleginnen erfahren, die Ute Gerhard als Herausgeberin von Büchern und Zeitschriften oder als Projektleiterin kennen lernten. Unbedachte Grammatik, Syntax oder Zeichensetzung und ein nachlässiger Stil können die Vermittlung wichtiger Gedanken und wissenschaftlicher Aussagen ebenso stören wie falsche Noten oder ein schiefer Ton die Interpretation musikalischer Werke verstellen. Ute Gerhard hat als passionierte, praktizierende Musikerin Kompetenzen wie die Fähigkeit zum konzentrierten Zuhören und zur Kooperation im Quartett ausgebildet, die keineswegs wissenschaftsferne Kompetenzen sind, ganz im Gegenteil.

\section{Gleichheit und Differenz}

Das Jahrhundert des Feminismus wurde von Ute Gerhard in vielen Studien nicht nur als Erfolgsgeschichte thematisiert, sie mahnte vielmehr immer wieder die "Vollendung des politischen Projekts der Moderne, die Einlösung des Versprechens der Freiheit und Gleichheit aller Menschen, auch der Frauen" an (Gerhard 2001, 35). ${ }^{3}$ Auch die Konferenzbeiträge

2 Vgl. dazu detailliert die homepage des Cornelia Goethe Centrums, http://www.uni-frankfurt. $\mathrm{de} / \mathrm{cgc}$.

3. Im Folgenden werden wir nur exemplarisch auf einzelne Arbeiten von Ute Gerhard verweisen. Eine vollständige Bibliografie ist auf der homepage des Cornelia Goethe Centrums einzusehen; vgl. http://www.uni-frankfurt.de/cgc. 
zeigen deutlich, dass postmodernes und postfeministisches Denken die weltweiten Ungerechtigkeiten und Ungleichheiten in den Geschlechterverhältnissen und die Verletzungen von Freiheit und Würde von Frauen bisweilen etwas zu voreilig ausblendet. Bei der Dekonstruktion der Geschlechterdifferenz und der Problematisierung der Kategorie ,Frauk als Identitätskategorie muss es vielmehr "um die kritische Reflexion über die Grundlagen feministischer Theoriebildung und über nichtintendierte politische Konsequenzen" gehen, wie Ute Gerhard in einem neueren Beitrag betont. Dabei bezieht sie sich auf das immer wieder thematisierte Risiko, dass mit einem feministischen "Wir" der Ausschluss und die mögliche Diskriminierung anderer verbunden sein kann (Gerhard 2001, 34).

Ute Gerhard vermittelt uns immer von Neuem den notwendigen Blick zurück auf die Geschichte des Feminismus, damit wir nicht geschichtsblind werden für die Zukunft. Die nach wie vor aktuelle Frage, die sie vor über 25 Jahren in ihrer Dissertation Verhältnisse und Verhinderungen formulierte, war nur möglich auf dem Hintergrund ihrer gesellschaftstheoretisch und historisch informierten Auseinandersetzung mit Konstruktionen der $\mathrm{Ge}-$ schlechterdifferenz, den Ursachen der Geschlechterungleichheit, insbesondere im Recht, und den unterschiedlichen Perspektiven der alten Frauenbewegung im Hinblick auf ihre jeweiligen selbstgesetzten Kulturaufgaben:

"Die sneue Frauenbewegung hat sich ebenfalls einer Kulturaufgabe gestellt, d.h. sie versucht, neue Beurusstseins- und Verhaltensformen zu entwickeln. Ihr Problem allerdings bleibt die theoretische und praktische Vermittlung ihrer Entwürfe mit den Widerständen der gesellschaftlichen Realität. Gelingt diese Vermittlung nicht, so bleibt nur der schmale Grat zwischen Mutterkult und weiblicher Gegenkultur, und der Protest der Frauen droht in
Anpassung oder folgenloser Verweigerung $z u$ verkommen." (Gerhard 1978, 189)

In diesem Zitat ist - wie unter einem Brennglas - ein Projekt vorgezeichnet, das zentriert ist um die Auseinandersetzung mit Gleichheit und Differenz. "Bis an die Wurzel des Übels" reichen ihre scharfsichtigen, gesellschaftstheoretisch orientierten Analysen des Rechts, um jene Vermittlung zu leisten, die in der zitierten Problemskizze von 1978 angesprochen war. In Bezug auf diese Praxis ist der Titel ihres viele Jahre später entstandenen Buches Atempause in gewisser Weise nur eine rhetorische Figur, die zu erneuter theoretischer Anstrengung und Reflexion des "Feminismus als demokratischem Projekt" verpflichtet und gleichzeitig zum gelassenen Blick auf die "Flaute" der Frauenbewegung ermuntert (Gerhard 1999). Ute Gerhard hat insbesondere mit ihrer Habilitationsschrift Gleichheit ohne Angleichung. Frauen im Recht (1990a) und ihrer Geschichte der deutschen Frauenbewegung - Unerhört (1990b) - gegen den - nicht zuletzt durch den Faschismus bedingten Erinnerungsverlust der Frauenverbände der Nachkriegszeit und die damit verbundene Geschichtsblindheit der neuen Frauenbewegung argumentiert. Die Töchter der Trümmerfrauen hatten mehr als nur den ideologischen Schutt der jüngsten Geschichte der Barbarei wegzuräumen. Es ging auch darum, einen neuen Blick auf die widersprüchliche Moderne mit ihren nicht eingelösten Möglichkeiten zu werfen und emanzipatorische Traditionslinien der Rechtskämpfe und der Rechtskritik des radikalen Flügels der alten Frauenbewegung des 19. Jahrhunderts zu entdecken.

Die Arbeit an einem rechtstheoretischen Gleichheitsbegriff, der die falsche Alternative von Gleichheit oder Differenz überwindet, bildet den berühmten roten Faden, der fast alle Arbeiten von Ute Gerhard durchzieht und ihre weit ge- 
fächerten politischen Formen der öffentlichen Einmischung bestimmt. So ist ihre Tätigkeit als Wissenschaftlerin nicht zu trennen von ihren Aktivitäten innerhalb der Frauenbewegung und als politisch engagierte Bürgerin, die sich der Kritik bestehender Institutionen - auch der Wissenschaft - und deren Umbau und Neubau verschrieben hat. ${ }^{4}$ Dazu zählt auch ihr Engagement als Mitbegründerin der Feministischen Studien 1982 und ihre aktive Mitarbeit in diesem Projekt für ein ganzes Jahrzehnt. Innerhalb der neuen Frauenbewegung richtete sich Ute Gerhard gegen die eindimensionale Rechtsskepsis einerseits und gegen jede politikferne Politik der Differenz andererseits. Damit stand sie schon immer jenseits eines essentialistischen Politikverständnisses.

„Das Ernstnehmen, mehr noch: das ZurGeltung-Bringen der anderen Wertmaßstäbe von Frauen, Gleichberechtigung trotz aller Verschiedenheiten, also, Gleichheit in der Differenz<, beinhaltet ein neues Verständnis und eine veränderte Praxis der, Freiheit und Gleichheit, ist m. E. das noch nicht eingelöste Versprechen der Menschenrechte als Denkmöglichkeit humaner Verhältnisse. (Gerhard 1990a, 12)

Recht dient nicht nur bestimmten patriarchalen (und klassenspezifischen) Herrschaftsinteressen, die Ungleichbehandlung begründen und absichern, sondern es enthält auch die Elemente, die über die Ungleichheit hinausweisen, ja diese überschreiten. Ute Gerhards Zugang macht deutlich, dass die Dekonstruktion des Rechts allein nicht ausreicht, um Geschlechterasymmetrien sichtbar zu machen und $\mathrm{zu}$ verändern, sondern dass erst die feministische Rekonstruktion, also Reformulierung des universalen Gehalts des
Rechts geeignet ist,Veränderungen zu erwirken.

Die Janusköpfigkeit des Rechts durch feministische Rechtskritik aufzuzeigen, auch die "Voreingenommenheit der Jurisprudenz" akribisch zu dechiffrieren und zugleich die emanzipatorischen Möglichkeiten des Rechts zu denken, ist Gegenstand von Ute Gerhards nie versiegendem Bemühen und zugleich ihre innovative Kraft.

Gegenwärtig werden oftmals Missverständnisse sichtbar, wenn etwa mit einer falsch akzentuierten Alternative von Gleichheit oder Differenz die Möglichkeit feministischer Politik abgewiesen oder jedes universalistische Denken mit dem Hinweis zurückgewiesen wird, Differenzen seien nicht mitgedacht. Ute Gerhard hat demgegenüber die dialektische Figur Gleichheit in der Differenz immer wieder neu kontextualisiert, begründet und in ihrer Bedeutung für die Forderungen von Frauen nach zivilen, politischen und sozialen Rechten entfaltet. Die Vorstellung von Geschlechtergerechtigkeit kommt, so ihr Fazit, ohne den rechtsphilosophischen und politischen Begriff von Gleichheit und einen damit verbundenen Freiheitsbegriff nicht aus:

"Als ein in historischen Kämpfen ebenso umstrittener wie mit Bedeutungen gesättigter Rechtsbegriff ist Gleichheit also kein absolutes Prinzip oder feststehendes Maß, vielmehr ein dynamisches Konzept, das in die , Geschichtlichkeit der Geschlechterdifferenz‘ eingebunden ist. Gleichheit entsteht als Problem nur, insofern es Differenzen zwischen den Individuen gibt, und es kann bei diesem Rechts-Begriff nur um Gleichheit in bestimmter, für die Lebenschancen relevanter Hinsicht handeln, niemals um Identität oder Angleichung. Die neuen Frauenbewegungen haben immer wieder

4 Vgl. beispielsweise ihr Engagement im Komitee für Grundrechte und Demokratie seit 1979; ihre Mitarbeit in der Petitionsinitiative zur Finanzierung von Frauenhäusern in der BRD 1983, die streitbaren Einmischungen in der Kontroverse um die Neuformulierung des $₫ 218$, ihre Mitarbeit in der Initiative "Frauen für eine neue Verfassung" und vieles mehr. 
um diese relevanten Hinsichten der Gleichheit angesichts von Differenzen, der Geschlechterdifferenzen wie der Unterschiede unter Frauen und anderer nicht repräsentierter Gruppen, gerungen und damit neue Standards für Gerechtigkeit gesetzt. «(Gerhard 2004,18, vgl. dazu detailliert Gerhard 1990a, 1999, 2003b).

Bedeutsam ist die historische Perspektive, die Ute Gerhards Werk auszeichnet. Sie ermöglicht es nicht nur, die Frauenbewegung in der Perspektive einer longue durée zu beschreiben, sondern auch ihre Traditionslinien und unerledigten, gegenwärtigen und zukünftigen Anliegen zu identifizieren $(1999,12 \mathrm{ff}$.).

\section{Frauenrechte sind Menschenrechte}

Seit der Weltfrauenkonferenz in Peking im Jahre 1995 ist zuvor durchaus Anstößiges und Unübliches üblich geworden, d. h. es wurde möglich, von Frauenrechten als Menschenrechten zu sprechen. Bei der Auseinandersetzung mit Rechtstheorien und Rechtstatsachen bezieht sich Ute Gerhard auf konkrete Unrechtserfahrungen von Frauen. Denn Unrechtserfahrungen ${ }^{5}$ sind der methodische Schlüssel, mit dessen Hilfe sich ein neuer Blick auf die Sozial- und Rechtsgeschichte, auf Verhältnisse und Verhinderungen, unter denen Frauen leben und arbeiten, erschließt. Damit reale Verhältnisse bewusst als Unrecht wahrgenommen werden können, bedarf es einer Blickrichtung, die bisherige Selbstverständlichkeiten erschüttert und deren Prämissen hinterfragt.

So hat Ute Gerhard die Verhältnisse im Preußen des frühen 19. Jahrhunderts durchleuchtet, um darzulegen, wie sich in dieser Epoche nach 1830 ein spezifisch neuer, nämlich bürgerlicher Patriarchalismus auf die Lage der Frauen auswirkte und in krassen Widersprüchlichkeiten im Hinblick auf die Rechte der Frauen niederschlug. Zwar hat die feministische Spurensuche dokumentiert, dass es Frauen in allen Epochen verstanden haben, ihre Stimme zu Gehör zu bringen und eigene Lebens- und Wirkungsräume zu entwerfen. Das 19. Jahrhundert aber, in dem sich in den westeuropäischen Staaten und in den Vereinigten Staaten von Amerika die bürgerliche Gesellschaft herausbildete, hat in besonderem Maße Frauen zu Zeuginnen von Unrechtserfahrungen - nicht nur von Frauen - gemacht. Aus ihrem Engagement für neue, menschenwürdigere Verhältnisse, z.B. in der Bewegung zur Abschaffung der Sklaverei, und aus der Enttäuschung darüber, dass ihnen als Frauen in dieser modernen Gesellschaft keine Stimme zukommen sollte, erwuchs ihr neues politisches Bewusstsein.

Von hervorragender Bedeutung in Deutschland ist hier die Frauen-Zeitung ${ }^{6}$ von Louise Otto, die ein Forum für die Unrechtserfahrungen von Frauen der verschiedensten Schichten bildete. Ute Gerhard beschreibt das 19. Jahrhundert als Anfang der Einmischung von Frauen in eine entstehende politische Öffentlichkeit und zitiert hierzu Louise Otto wie folgt:

"Wir Frauen fordern einfach unser Recht, unser Menschen-Recht (...) Wir aber wollen keine Puppen, sondern Menschen sein; Menschen, die ihre bestimmten Rechte und Pflichten haben, wie alle denkenden Wesen (...) Die Stellung, die wir in der Welt einnehmen, soll nach den Gesetzen der Natur und Sittlichkeit - was dasselbe ist - mithin unserer eigenen Kraft, sich richten. Dies ist es, was ich

5 Ute Gerhard hat sich kürzlich theoretisch mit kontroversen Ansätzen zum Erfahrungsbegriff auseinandergesetzt. Vgl. Feministische Studien 2/2001.

6 Ute Gerhard hat 1979 zusammen mit Elisabeth Hannover-Drück und Romina Schmitter die Frauen-Zeitung von Louise Otto unter dem Titel "Dem Reich der Freiheit werb' ich Bürgerinnen" neu herausgegeben und kommentiert. 
,Frauen-Recht genannt habe und wovon wir gegenwärtig noch ziemlich weit entfernt sind". (Luise Otto, "Die Freiheit ist untheilbar", zit. in Gerhard 1997, 524).

Ähnlich den Frauen in der Französischen Revolution, die wie Marie Aubray, genannt Olympe de Gouges, am 7. September 1791 eine (von Ute Gerhard neu übersetzte) eigene "Erklärung der Rechte der Frau und Bürgerin« der Erklärung der Menschen- und Bürgerrechte vom 26. August 1789 entgegenstellte, sahen sich ein halbes Jahrhundert später auch die Frauen in den deutschen Ländern unversehens aus den Verhandlungen über die neue Verfassung und die "Grundrechte der Deutschen" in der Paulskirche ausgeschlossen. Louise Ottos einfacher Lehrsatz, dass Freiheit unteilbar sei, blieb "unerhört", ganz unabhängig davon, dass mit dem Scheitern der Revolution und der Ablehnung der Paulskirchenverfassung durch den preußischen König auch für die männlichen Staatsbürger die Ansprüche auf bürgerliche Rechte und Freiheiten sowie politische Rechte ein weiteres halbes Jahrhundert zu warten hatten (Gerhard 1990b).

Aufschlussreich ist in einer historischen Perspektive die Bedeutung des Vereinsrechts zur Niederhaltung und zugleich zur Ausübung von Frauenansprüchen (vgl. Gerhard 1978, 1997). Bis zum Jahre 1908 blieb Frauen in Deutschland jegliche politische Betätigung grundsätzlich untersagt. Damit war den Frauen der Weg zur Mitgestaltung eines Gemeinwesens nach neuen liberal-bürgerlichen oder gar demokratischen Werten verstellt. Sie reagierten unter anderem mit Ironie oder Sarkasmus, wie beispielsweise Minna Zimmermann in der Frauen-Zeitung Nr. 35 aus dem Jahre 1850, die die Barrieren gegen
Frauenberufstätigkeit außerhalb des Proletariats aufspießte:

»Wenn die Menschen gewohnt wären, ihre Handlungen in Worte zu übersetzen, so wïrden wir etwa folgendes Gespräch zwischen einer Mutter und ihrer Tochter vernehmen. Die Mutter beginnt: Mein Kind, du bist nun envachsen, wohlunterrichtet und eingesegnet, was wirst du jetzt thun, um deinen LebensUnterhalt zu gewinnen und der Menschheit in deiner Thätigkeit dankbar den Zoll abzutragen, den jedes Wesen ihr schuldet? Hierauf erwidert die Tochter mit edlem Selbstgefühl: Ich werde gar nichts thun, sondern warten bis jemand kommt, der mir Nahrung und Kleidung reiche. Und die Mutter antwortet: Bravo, meine Tochter. Ich wollte dich nur prüfen, ob du unberüht vom Pesthauch radikaler Einfälle deine schöne Weiblichkeit bewahrt hast." (zit. in: Gerhard 1978, 477).

Neben solch unverhohlener Ironie und scharfer Kritik beschritten Frauen aber auch mit List und Geschick unverdrossen die Politikwege, die ihnen geblieben waren: Vereinstätigkeiten, die sie trotz aller Verbote für politische Betätigung dennoch versteckt nutzten, Petitionen und Demonstrationen. ${ }^{7}$ Unter diesen Bedingungen schuf sich die erste Frauenbewegung in Deutschland im Jahre 1865 den Allgemeinen Deutschen Frauenverein, dem es satzungsgemä $\beta$ darum ging, "den Kultureinfluss der Frau zu voller innerer Entfaltung und freier sozialer Wirksamkeit zu bringen" (Lange 1924, 18).

Die verschiedenen Strömungen der ersten Frauenbewegung in Deutschland rangen um Inhalt und Durchsetzungsform eben dieses "Kultureinflusses" der Frauen. Vor 20 Jahren hat Ute Gerhard in einem Aufsatz für die Zeitschrift Feministische Studien an die Auffassung der Juristin Anita Augspurg, führendes Mitglied

7 Betrachten wir die weltweite Situation heute, so finden sich übrigens wiederum Vereinstätigkeit, Petitionen, Konferenzen und Demonstrationen als zentrale Mittel, mit deren Hilfe sich Frauen Gehör zu schaffen versuchen. 
der Radikalen der ersten Frauenbewegung, erinnert. Augspurg äußerte sich im Jahre 1895 mit folgender These: "Die Frauenfrage ist zwar zum großen Teil Nahrungsfrage, aber vielleicht in noch höherem Maße Kulturfrage, (...) in allererster Linie aber ist sie Rechtsfrage, weil nur von der Grundlage verbürgter Rechte (...) an ihre sichere Lösung überhaupt gedacht werden kann". "Rechtsfrage" bedeutet in dieser Sicht die Anerkennung des unveräußerlichen Rechts, zusammen mit anderen erwachsenen Menschen in einem Gemeinwesen gleichberechtigt die eigene Stimme zur Geltung zu bringen (vgl. Gerhard/Schlüpmann 1984).

Der Mainstream der deutschen Frauenbewegung folgte damals dieser Auffassung nicht. Eine menschenrechtliche Begründung für die staatsbürgerschaftliche Gleichstellung von Frauen hielt Helene Lange zwar für die amerikanischen Verhältnisse angemessen, nicht aber für die deutschen. Lange sah die leitende Idee der Frauenbewegung in einer "Weiblichkeit als Kulturform" (Lange 1924, 145). Es ging ihr um die "positive eigenartige Leistung, die Frauen, und nur sie, zur Gestaltung der Kulturwelt beitragen können" und die dadurch »den Frauen dauernd ihren Platz im öffentlichen Leben sichern" kann (ebd.). Geschlechterdemokratie als verpflichtender Qualitätsmaßstab für die Güte staatlicher Verfassung und Verfassungspraxis oder - wie Louise Otto es formuliert hatte - als "Barometer der Staaten" war damit nicht gemeint (Gerhard 1999, 15). Das in diesen differenten Rechtsauffassungen zum Ausdruck kommende konfliktive Verständnis von Frauenidentitäten, weiblichen Kulturen und frauenpolitischen Zielen durchzieht noch heute die verschiedenen Strömungen organisierter Frauen weltweit.

Wird mit der "Kulturfrage" die normative Frage angesprochen, wie das $\mathrm{Zu}$ sammenleben von Menschen angesichts ihrer tatsächlichen Verschiedenheiten und angesichts des unveräußerlichen Rechts auf Gleichheit gestaltet werden soll - und so verstehen wir Anita Augspurg und Ute Gerhard -, so stellt sich damit im Kern die Frage nach der politischen Gestaltung fürsorglicher Praxis in sich modernisierenden Gesellschaften.

\section{Politische Gestaltung fürsorglicher Praxis}

Sorge für andere, und zwar unabhängig von Verpflichtungen, die mit der Institution der Ehe verbunden waren, gehört seit langem zu den Lebensformen von Frauen. Der besondere Charakter frauenbewegter öffentlicher Aktivitäten im Entstehungsprozess der bürgerlichen Gesellschaft rührt genau daher: Wo auch immer aktive Frauen ihrer sozialen Herkunft nach verankert sein mochten, der Horizont ihrer politischen und sozialen Anteilnahme überschritt den beschränkten Gesichtskreis des eigenen sozialen Milieus. Ute Gerhard und Heide Schlüpmann haben in dem erwähnten Themenheft der Feministischen Studien (1984) über die Radikalen in der alten Frauenbewegung gezeigt, dass die grundlegende Forderung nach Rechtsgleichheit bei Helene Stöcker, Anita Augspurg und vielen anderen zugleich mit einer Politik für Mutterschutz, Arbeiterinnenschutz und Rechtskritik im Hinblick auf doppelte Moral einherging. Die Entwicklung des organisierten Sozialstaats in Deutschland, der in unseren Tagen völlig neue Konturen erhält, wäre seinerzeit ohne die Prägung und Mitwirkung sozialpolitisch engagierter Frauen undenkbar gewesen.

Diese Seite der Frauenbewegung ihre Bedeutung als normative oder moralische Sachwalterin (entrepreneurin) - ist keineswegs zufällig auch international wirksam geworden, so in der Bewegung zur 
Abschaffung der Sklaverei in den USA und im Kampf gegen Frauenhandel (im Sprachgebrauch der damaligen Zeit als "weißer Sklavenhandel" bezeichnet) - bis hin zum friedenspolitischen Plädoyer, auch im Verkehr der Staaten untereinander dem Prinzip des Rechts Vorrang zu verschaffen. Woodrow Wilsons 14 Punkte am Ende des Ersten Weltkriegs basierten bekanntlich weitgehend auf den 10 Punkten, die vom Internationalen Frauenausschuss für dauerhaften Frieden aufgestellt wurden. Deren Folgeorganisation, die Internationale Frauenliga für Frieden und Freiheit, stellte in ihrer Note an die Friedenskonferenz in Versailles zwar »mit Genugtuung fest", dass der Gedanke eines Völkerbunds allgemeine Anerkennung gefunden habe, bemängelte aber, dass in der vorgelegten Völkerbundsatzung wesentliche Bedingungen "zur Sicherung des Weltfriedens« fehlten, etwa die Aufnahme aller Staaten, "die es wünschen und die sich bereit erklären, die Pflichten der Mitgliedschaft zu erfüllen" und vor allem die Aufstellung von Grundsätzen in allen Ländern zur Gleichstellung der Frauen mit den Männern »in den gesetzgebenden und Verwaltungs-Körperschaften, sowohl den internationalen wie den nationalen«. Dringend wurde die Aufnahme des Grundsatzes der Beachtung der vollen persönlichen und bürgerlichen Rechte der Frauen, insbesondere des Stimmrechts der Frauen, in die Völkerbundssatzung gefordert (Brinker-Gabler 1980, 248). Ute Gerhard hat diese internationalen Dimensionen von Frauenpolitik immer wieder in ihre Überlegungen einbezogen:

"Unter dem Motto sfür die Gewalt des Rechts gegen das Recht der Gewalt - hatte die Frauenrechts- und Friedensbewegung, unterstützt von Bertha von Suttner, bereits 1899 anlässlich der Ersten Haager Friedenskonferenz die Frauen aller Länder zur Intervention aufgerufen und Millionen Unterschriften zu- sammen gebracht. Heute gewinnt diese Forderung für Frauen in allen, auch den anderen Teilen der Welt neue Aktualität«. (Gerhard 2004)

Ute Gerhard geht es in ihrer Rechtskritik nicht um die "Perpetuierung des Geschlechterkampfes um das richtige Recht« und ebenso wenig um eine »Politik des Unterschieds". Vielmehr hat sie das "Ernstnehmen, mehr noch: das ZurGeltung-Bringen der anderen Wertmaßstäbe" und Erfahrungen von Frauen auf die Tagesordnung gesetzt (Gerhard 1990a, 12). Diese Perspektive steht auch hinter ihrer Auseinandersetzung mit klassischen Staats- und Vertragstheorien in Gleichheit ohne Angleichung (1990a).

Erst der feministischen Kritik an der politischen Philosophie der Aufklärung fiel auf, dass sich im klassischen Konstrukt des Gesellschaftsvertrags die Bürgerrechte auf Männerrechte reduzierten und unvermeidliche, existenzielle Abhängigkeitssituationen im Leben jedes Menschen ausgeblendet wurden. Die Selbstverständlichkeit, mit der aus dem neuen Diskurs über Unabhängigkeit, Freiheit und Gleichheit in der bürgerlichen Gesellschaft alles herausfiel, was auf die Entwicklungsphasen von Kindheit und Alter in ihrer Bedürftigkeit und Abhängigkeit von der $\mathrm{Zu}$ wendung anderer Menschen hätte hinweisen können, ist von feministischen Theoretikerinnen mit den Konzeptionen des Patriarchalismus und eines impliziten Geschlechtervertrages exklärt worden. Tatsächlich ging die philosophische und rechtstatsächliche Herausbildung des bürgerlichen Individuums mit der Familiarisierung der (verheirateten) Frauen zusammen, eine Entwicklung, die Ute Gerhard (1990a) besonders für Deutschland rechtshistorisch im Detail untersucht hat. Sie hat in ihrer Kritik an historischen Darstellungen mit allgemeinem Anspruch immer wieder darauf hingewiesen, dass sich für Frauen die historische Abfolge 
der Erweiterung ihrer Rechte anders darstellte als für Männer: Nicht bürgerliche Rechte, sondern politische Beteiligungsrechte standen bei Frauen am Anfang ihrer politischen Bestrebungen.

\section{Perspektiven in einer zerklïfteten Welt}

Der größte Teil der Menschen lebt heute nicht in postindustriellen Gesellschaften, sondern in vorindustriellen oder in peripher-kapitalistischen bzw. Transformationsgesellschaften. Die Strukturen, innerhalb derer heute die Aufgaben der politischen Organisation des innergesellschaftlichen Zusammenhalts gelöst werden müssen, sind demzufolge höchst unterschiedlich. Ute Gerhard hat sich im Rahmen verschiedener Netzwerke und Forschungsvorhaben dieser Heterogenität angenommen, sei es im Rahmen vergleichender Untersuchungen in der EU, sei es durch Forschung und Konferenzen zu Geschlechterordnungen außerhalb Europas. Zweifellos steht das universale Moment im Projekt der Frauenbewegungen vor schwierigen Herausforderungen. ${ }^{8}$

Wie in früheren Zeiten schlagen Frauen sehr verschiedene Wege ein, um vor Ort und in den bestehenden internationalen Strukturen wirksam zu werden. Im Rahmen der UNO und der von ihr initiierten Weltkonferenzen zu globalen Themen wie der Erhaltung von Lebensgrundlagen, der Durchsetzung von Menschenrechten, der Verbesserung von sozialen Situationen usw. haben organisierte Frauen es verstanden, die Vielfalt und die internen Konfliktlinien unter Frauen in richtungweisende Ansätze für politisches Handeln zu transformieren. Im Rahmen einzelner UNO-Son- derorganisationen, wie insbesondere der Internationalen Arbeitsorganisation, ist es gelungen, ein Engagement der Organisationsspitze für die grundlegende Beachtung von Geschlechterverhältnissen und ihrer Verbesserung zu gewinnen. Das reicht von der Unterstützung geschlechtersensibler Beschäftigungsanalysen in der informellen Ökonomie bis hin zur Unterstützung von Programmen, die zur Stärkung der Handlungsfähigkeit von Frauen in lokalen Ökonomien beitragen sollen. Stichworte sind hier, ganz analog zur früheren Geschichte »empowerment", "capacity building" etc. Solche Ansätze würden völlig ins Leere laufen, wenn nicht vielfältige Frauenaktivitäten bestünden, die jeweils an ihrem Ort gegen ungerechte Geschlechter- und damit Gesamtverhältnisse kämpften.

Keine Gesellschaft, kein Gemeinwesen, keine Kultur kann sich heute der Auseinandersetzung über die Geschlechterordnung entziehen. Positionen, die Frauen kein aktives oder doch wenigstens passives Wahlrecht zubilligen, die Frauen also nicht die gleichen bürgerlichen Rechte wie Männern zugestehen wollen, gibt es noch genügend. Aber zu Beginn des 21. Jahrhunderts müssen sie mit sehr viel stärkeren Gegenpositionen rechnen. Die Forderung nach gleichen Rechten erweist sich damals wie heute in Gestalt von Kulturkonflikten als Kern der Ordnungskonflikte (vgl. Gerhard 2003b). Ob Frauen wie Männern eine unabhängige individuelle Existenz zugeschrieben wird, und wie die Verhältnisse zwischen den Geschlechtern und den Generationen gedacht werden - hierarchisch und exklusiv oder gleichberechtigt und inklusiv - entscheidet sich in diesem Konflikt.

"Selbst die 1979 von der UNO-Generalversammlung verabschiedete >Konvention über

8 Vgl. exemplarisch die Debatte um (europäische) Bürgerrechte, die aus verschiedenen Perspektiven von Ute Gerhard und Vlasta Jalusić in Feministische Studien 1/2003 gefuhrt wurde. 
die Beseitigung jeder Form von Diskriminierung der Frau (CEDAW) wurde von vielen Staaten nur unter Vorbehalt ratifiziert (...). Trotzdem, diese Rechtsform verleiht denjenigen eine Stimme, die Unrecht erfahren haben. Es sind Rechtsverbürgungen jenseits der jeweils geltenden staatlichen Gesetze, die von jeder einzelnen in Anspruch genommen werden können und ihre Unrechtserfahrungen in die Sprache des Rechts übersetzen. "(Gerhard 2004; vgl. 2003b).

Für Ute Gerhard ist gegenwärtig noch nicht abzusehen, ob eine "dritte Welle» des Feminismus als Menschen- und Frauenrechtsbewegung in die Geschichte eingehen wird. Rechte sind kein "Haben oder Besitz, sondern müssen immer wieder erkämpft, verteidigt und an den geltenden Standards von Gerechtigkeit neu vermessen werden. Die Verleihung des Friedensnobelpreises 2003 an die iranische Anwältin der Menschenrechte Shirin Ebadi könnte ein Signal und eine Ermutigung zum Aufbruch sein" (Gerhard 2004; vgl. $2003 \mathrm{~b}$ ). Allerdings sind auch Einwände zu antizipieren, nicht zuletzt von islamischen AktivistInnen hierzulande, die den Universalismusanspruch von Frauen- und Menschenrechten in Frage stellen und eine Kontextualisierung und Relativierung fordern (Gerhard 2003b). Gehören die Frauen- und Menschenrechte auch zu den großen eurozentrischen Erzählungen der Moderne, von denen wir uns verabschieden müssen, weil sie von den vielen kleinen postmodernen Erzählungen, postkolonialen Wissensformen oder religiösen Überzeugungen durchkreuzt werden? Und wie wäre ein feministisch verstandener Universalismus denkbar, wenn Anerkennungsansprüche von Differenzen unterschiedlichster Art vielleicht nur noch behauptet werden, sich aber auf kein geteiltes Verständnis von gleicher Achtung und moralischer Autonomie mehr beziehen lassen? Gerade aus einer feministischen Perspektive wären - nicht nur im
Kontext islamischer Debatten - gegen jede politisierte Theologie und jeden theologischen Kern von Politik, die Freiheit, die Menschen- und Frauenrechte konsequent $\mathrm{zu}$ verteidigen (Gerhard 2003c).

In merkwürdigem Gegensatz zu ihren männlichen und weiblichen Kritikern hatten Frauen in den verschiedenen Frauenbewegungen immer fest im Blick, was für Ute Gerhard von großer Relevanz ist: dass ihre Bestrebungen um Freiheit nicht nur Abhängigkeiten beenden sollten, sondern auch Freiheiten für die Gestaltung des Zusammenlebens zuischen Menschen überhaupt beinhalteten. In allen Kontexten bietet der Feminismus politischen Gemeinwesen Maßstäbe für das von Helene Stöcker formulierte "Glück gegenseitiger Anerkennung" (Gerhard 1991) und eine "soziale Praxis der Anteilnahme" (Gerhard 2003a).

Das Jahrhundert des Feminismus - so der Tenor des Symposiums für Ute Gerhard - ist unabgeschlossen: Es ist ein Projekt mit verschiedenen Traditionssträngen und Brüchen, gekennzeichnet durch vielfältiges Ringen um seine Ausformung, aber auch durch bahnbrechende Innovationen. Es ist ein Projekt, dessen Versprechungen noch längst nicht eingelöst sind.

\section{Literatur}

Brinker-Gabler, Gisela (Hrsg.) (1980): Frauen gegen den Krieg, Frankfurt am Main

Gerhard, Ute (2004): "Das Jahrhundert der Feminismen. Konzepte und Streitpunkte". (Ms., erscheint in französischer Sprache in einem von Brigitte Studer/Françoise Thébaud/Florence Rochefort/Michèle Zancarini/Eliane Gubin/Catherine Jacques herausgegebenen Sammelband: Le siècle des Feminismes, Paris)

- zusammen mit Trudie Knijn und Anja Weckwert (Hrsg.) (2003a): Enwerbstätige Mütter. Ein europäischer Vergleich. München 
- (2003b): "Menschenrechte sind Frauenrechte. Überlegungen zu Gleichheit und Geschlechtergerechtigkeit im Islam." In: Mechthild Rumpf/Ute Gerhard/Mechtild M. Jansen (Hrsg.) Facetten islamischer Welten. Geschlechterordnungen, Frauen- und Menschenrechte in der Diskussion. Bielefeld

- zusammen mit Mechthild Rumpf (Hrsg.) (2003c): Streitpunkte in islamischen Kontexten. Feministische Studien 2

- (2001): "Frauenbewegung - Frauenforschung - Frauenpolitik. Innovation und Selbstreflexion«. In: Ursula Hornung/Sedef Gümen/Sabine Weilandt (Hrsg.): Zwischen Emanzipationsvision und Gesellschaftskritik, Forum Frauenforschung Bd. 14, Münster

- (Hrsg.) (2000): Dokumentation der Namensgebung am 7. Dezember 2000. Mitteilungen des Cornelia Goethe Centrums für Frauenstudien, Frankfurt/M.

- (1999): Atempause. Feminismus als demokratisches Projekt. Frankfurt/M.

- (Hrsg.) (1997): Frauen in der Geschichte des Rechts.Von der Frühen Neuzeit bis zur Gegenwart. München

- (1993): mFern von jedem Suffragettentum - Frauenpolitik nach 1945, eine Bewegung der Frauen?". In: Ulla Wischermann/Elke Schüller/Ute Gerhard (Hrsg.): Staatsbürgerinnen zwischen Partei und Bewegung. Frankfurt am Main

- zusammen mit Eva Senghaas-Knobloch (Hrsg.) (1992): Umbruch in Europa, Aufbruch der Frauen?, Feministische Studien 2

- (1991): "Maßstäbe einer Verfassung auch für Frauen - eine andere Freiheit, Gleichheit, Würde". In: Claudia Burgsmüller/Mo- nika Frommel/Ute Gerhard/Birgit Laubach/Andrea Maihofer/Regine Walch (Hrsg): Frauen für eine neue Verfassung. Feministische Studien extra-Heft

- (1990a): Gleichheit ohne Angleichung. Frauen im Recht. München

- (1990b): Unerhört. Die Geschichte der deutschen Frauenbewegung, unter Mitarbeit von Ulla Wischermann. Reinbek

- (1990c): "Bürgerliches Recht und Patriarchat". In: Ute Gerhard/Mechtild Jansen/Andrea Maihofer/Pia Schmid/Irmgard Schultz (Hrsg.): Differenz und Gleichheit. Menschenrechte haben (k)ein Geschlecht. Frankfurt/M.

- zusammen mit Jutta Limbach (Hrsg.) (1988): Rechtsalltag von Frauen. Frankfurt/M.

- zusammen mit Heide Schlüpmann (Hrsg.) (1984): Die Radikalen in der alten Frauenbewegung. Feministische Studien 1

- zusammen mit Elisabeth Hannover-Drück und Romina Schmitter (Hrsg.) (1979): "Dem Reich der Freiheit werb' ich Bürgerinnen ". Die Frauen-Zeitung von Louise Otto. Frankfurt/M.

- (1978, $\left.{ }^{4} 1989\right)$ : Verhältnisse und Verhinderungen. Frauenarbeit, Familie und Rechte der Frauen im 19. Jahrhundert. Frankfurt/M.

Lange, Helene $\left(1908,{ }^{3} 1924\right)$ : Die Frauenbewegung und ihre modernen Probleme. Berlin

Senghaas-Knobloch, Eva (2004): »Vom Glück gegenseitiger Anerkennung. Das Jahrhundert des Feminismus: Menschenrechte und fürsorgliche Praxis. Für Ute Gerhard zum 65. Geburtstag". In: Frankfurter Rundschau. Dokumentation. Nr. 46, 24. 2. 2004, S. 7 